\title{
A UTILIZAÇÃO MEDICINAL DO CANABIDIOL COMO RECURSO TERAPÊUTICO: REVISÃO BIBLIOGRÁFICA
}

\author{
THE MEDICINAL USE OF CANNABIDIOL AS THERAPEUTIC RESOURCE: REVIEW
}

DOI: $10.16891 / 2317-434 X . v 8 . e 2 . a 2020 . p p 581-590$

Recebido em: 04.03.2020 | Aceito em: 15.04.2020

Francisco Eduardo Ferreira Alves

\author{
Universidade Estadual da Paraíba \\ E-mail: fcoeduardoferreira@hotmail.com
}

\section{RESUMO}

A Cannabis sativa possui cerca de 60 tipos de canabinóides, dentre os quais o tetrahidrocanabinol (THC) e o canabidiol (CDB) apresentam finalidade terapêutica. O THC, por sua vez, possui propriedades que geram efeitos cognitivos e psicológicos, promovendo conflitos de interesse na condição de comercialização. O canabidiol vem recebendo expressiva atenção na comunidade científica e médica. O estudo objetivou identificar, a partir de evidências científicas, as características da utilização medicinal do canabidiol como recurso terapêutico. Trata-se de uma revisão de literatura com agrupamento dos dados e síntese do conhecimento acerca da temática, para a busca, foram utilizadas as bases acadêmicas: Medical Literature Analysis and Retrieval System on-line (MEDLINE), Literatura Latino-Americana em Ciências de Saúde (LILACS), Scientific Electronic Library Online (SciELO), usando os descritores Canabidiol Terapêutico canabidiol, Toxicidade. Os resultados apontam que o canabidiol é uma substância promissora, caracterizada por exercer seus efeitos farmacológicos por uma diversidade de mecanismos de ação, foi recentemente excluída da lista de substâncias não prescritas pela Agência Nacional de Vigilância Sanitária (ANVISA). Observaram-se algumas aplicações terapêuticas, como antipsicótico, ansiolítico, antidepressivo e em diversas outras condições com resultados satisfatórios, formulados à base de Cannabis sp. e seus derivados disponíveis no mercado. Por conseguinte, algumas limitações devem ser enfrentadas, especialmente porque outros canabinódes e a própria Cannabis sativa continuam proscritos no Brasil, uma vez que o uso indiscriminado dessas substâncias pode causar intoxicação e dependência, Com a atual revisão, percebe-se que, o foco dos estudos clínicos em humanos deve ser realizado, com período maior de observação dos efeitos em tratamentos prolongados, assim como analisado os efeitos adversos ao uso do CDB.

Palavras-chave: Canabidiol; Terapêutico canabidiol; Toxicidade; Tratamento.

\section{ABSTRACT}

Cannabis sativa has 60 types of cannabinoids, among which two stand out for therapeutic purposes: tetrahydrocannabinol (THC) and cannabidiol (CBD). THC, despite its medicinal effects, has cognitive and psychological effects, which provides a barrier to its use. However, Cannabidiol has received significant attention in medicine. This is because of its benefits in several pathologies and not causing, in principle, chemical dependency, because the studies were inconclusive about it. Thus, this study aimed to identify, from scientific evidence, the characteristics of the medicinal use of cannabidiol as a therapeutic resource. The literature review was adopted as data grouping method and synthesis of knowledge about the theme, in which the following academic databases were used: Medical Literature Analysis and Retrieval System Online (MEDLINE), Latin American and Caribbean Health Sciences Literature (LILACS), Scientific Electronic Library Online (SciELO) and Google Scholar. The results shows that it is a promising substance for the treatment of a variety of diseases and recently deleted from the list of prohibited substances by the National Health Surveillance Agency. Some therapeutic applications were observed, such as antipsychotic, anxiolytic, antidepressant and in several other conditions with satisfactory results, formulated based on Cannabis sp. and their derivatives available on the market. Therefore, some limitations must be faced, especially since other cannabinodes and Cannabis sativa itself are still banned in Brazil, since the indiscriminate use of these substances can cause intoxication and dependence. With the current review, it is clear that the focus of human clinical studies should be carried out, with a longer period of observation of the effects in prolonged treatments, as well as analyzing the adverse effects to the use of the CDB.

Keyword: Cannabidiol. Therapeutic use of cannabidiol. Toxicity, Treatment. 


\section{INTRODUÇÃO}

Historicamente, no Brasil, o uso de ervas e substâncias com ações calmantes, analgésicas e outros efeitos já se fazia presente no cotidiano de vida dos brasileiros. Apesar dos avanços em pesquisas que demostram a efetividade do uso de tais substâncias, algumas, como a C. sativa, conhecida como maconha, ainda apresentam usos limitados. Essa substância pode ser utilizada com fins medicinais, correspondendo a práticas milenares (SILVA, et al., 2018).

O Cannabis é o gênero de uma planta popularmente conhecida no Brasil como maconha, originária da Ásia e pertencente à família Cannabaceae, cujas subespécies mais notórias são a Cannabis sativa subespécie sativa, a Cannabis sativa subespécie indica e a Cannabis sativa subespécie ruderalis (MATOS et al., 2017). A maconha, no século XIX, não era considerada nociva, pois sua comercialização era de fácil acesso em farmácias, produtos que possuíam substâncias canabinoides, como anti-inflamatórios e remédios relacionados a problemas respiratórios. (SILVA et al., 2018).

A expectativa com relação ao uso do CBD como opção terapêutica para transtornos psiquiátricos deve-se à ausência de seus efeitos psicoativos e na cognição, segurança, boa tolerabilidade, ensaios clínicos com resultados positivos e o amplo espectro de ações farmacológicas (PINTO; CIPRIANO, 2015). Ademais, efeitos terapêuticos para doenças como artrite reumatoide, doença de Alzheimer e Parkinson, ansiedade, esquizofrenia e depressão são identificados na erva. Entretanto, o uso abusivo pode apresentar diversos efeitos colaterais, como taquicardia, secura da boca e olhos avermelhados (OLIVEIRA; LIMA, 2016).

O CBD é uma substância que atualmente é de grande interesse científico e acadêmico, destacando-se por apresentar efeitos benéficos para diversas doenças e transtornos que necessitam de várias opções farmacológicas para seu combate, podendo reduzir o sofrimento de muitas pessoas que fazem uso de medicamentos convencionais e tradicionais. Sabe-se que o CBD apresenta baixa propensão a produzir efeitos colaterais, comparando-se com alguns medicamentos utilizados para a mesma finalidade.

Devido à regulamentação administrativa, os indivíduos com recomendação médica de uso do CBD permanecem tendo que recorrer ao Poder Judiciário para obter o fármaco para tratamento das enfermidades que os acometem, tendo em vista que o Estado permanece negando o fornecimento (MELO e SANTOS, 2016).

Desse modo, é de grande relevância o uso da C. sativa para fins medicinais, assim como alvo de maiores pesquisa, uma vez que, evidenciado potencial farmacológico de alguns de seus princípios ativos, estudos acerca destas substâncias definam melhores estratégias de uso, tanto em relação à dose quanto à frequência e cuidados acerca de possíveis relações (GOTIJO et al., 2016.

A relevância ímpar dessa temática e da importância do uso racional e adequado do CBD estimula a caracterização sobre a utilização medicinal do canabidiol como agente terapêutico, bem como descrição das intoxicações, dependência e tolerância.

\section{METODOLOGIA}

Trata-se de uma revisão integrativa, com análise de dados e organização de informações encontradas em bancos de dados científico, utilizando revistas acadêmicas e artigos científicos disponíveis na internet.

A revisão bibliográfica foi adotada como método de agrupamento dos dados e síntese do conhecimento acerca da temática proposta, de modo a responder a seguinte questão norteadora: Quais são os principais tratamentos de enfermidades em que o canabidiol está inserido e o relevante papel das pesquisas científicas sobre esse princípio ativo no tocante ao uso como farmacoterapêutico.

Optou-se por selecionar estudos publicados no período temporal de 2015 a 2020, escritos nos idiomas inglês, espanhol e português. Foram utilizadas bases acadêmicas como: Medical Literature Analysisand Retrieval System on-line (MEDLINE), Literatura LatinoAmericana em Ciências de Saúde (LILACS), Scientific Electronic Library Online (SciELO).

Realizou-se a busca com descritores em Ciências da Saúde (DeCS) e controlados com as seguintes palavras-chave: "Canabidiol", "terapêutico canabidiol", "toxicidade" e "tratamento". Os manuscritos foram selecionados pelo título e resumo, sendo, posteriormente, avaliados na íntegra para uma completa apreciação do material retido, sendo os resultados apresentados de maneira descritiva.

Adotaram-se os seguintes critérios de inclusão: artigos publicados nos últimos cinco anos, até janeiro de 2020. Tais publicações foram organizadas em tabelas para análise. Estudos clínicos em humanos e não humanos, relacionados ao tratamento com o uso do Canbidiol, foram inclusos.

Foram excluídos estudos clínicos onde não se pode estabelecer através de seu uso terapêutico a dose utilizada no protocolo, como o uso da Cannabis sativa fumada e defumada, também foram excluídas publicações 
fora do tempo da pesquisa, assim como estudos clínicos com objetivos repetidos e estudo com animais apenas testando o princípio ativo, não relacionado ao tratamento.. A análise utilizada como critério dos artigos, foi a observação de suas metodologias de acordo com o objetivo proposto pelo artigo, assim como seus resultados obtidos.

Existe mais de 1.000 publicações com o descritor canabidiol, porem com uso para terapias medicinais e estudos clínicos esse número é reduzido a 74 artigos incluindo as três bases de dados descritas. Um grande número de artigos publicados nos últimos cinco anos, são artigos de revisão, ou seja, sem testes clínicos do uso da dose do canabidiol para fins de tratamento. Dentre os 74 artigos com eficácia clínica, foram selecionados

\section{RESULTADOS E DISCUSSÃO}

Na composição desse estudo, foram encontrados 74 artigos científicos, sendo selecionados 15 artigos, de acordo com os criterios de inclusõa e exclusçao. Na base de dados LILACS, foram obtidos 11 artigos. Na base de dados MEDLINE, foram obtidos 59 artigos na totalidade, e quatro artigos na SciELO.

Com a análise e leitura dos textos que norteavam a pesquisa dos 15 artigos, todos identificam a relevância da pesquisa em torno do CDB relacionado a tratamentos clínicos.

Tabela 1. Resultados dos artigos utilizados na pesquisa bibliográfica (2015-2020)

\begin{tabular}{|c|c|c|c|c|c|}
\hline Referência & Estudo & Protocolo & $\begin{array}{l}\text { Tempo } \\
\text { de Uso }\end{array}$ & $\begin{array}{l}\text { Efeitos } \\
\text { Adversos }\end{array}$ & Desfecho \\
\hline $\begin{array}{c}\text { CAPANO, } \\
2020 .\end{array}$ & $\begin{array}{l}\text { Evaluation of the effects } \\
\text { of CBD hemp extract on } \\
\text { opioid use and quality of } \\
\text { life indicators in chronic } \\
\text { pain patients: a } \\
\text { prospective cohort study }\end{array}$ & $\begin{array}{l}\text { CBD, } 0,5 \mathrm{mg} \\
\text { de THC, } 0,3 \\
\text { mg de } \\
\text { canabidivarina }\end{array}$ & $\begin{array}{l}\text { Uso } \\
\text { diário }\end{array}$ & Sem efeitos & $\begin{array}{l}\text { Pode ser usado para } \\
\text { controle da Dor crônica e } \\
\text { melhorar o sono. }\end{array}$ \\
\hline $\begin{array}{l}\text { GUIDA, } \\
2019\end{array}$ & $\begin{array}{l}\text { Cannabis medicinal como } \\
\text { recurso terapêutico: } \\
\text { estudo preliminar }\end{array}$ & $\begin{array}{c}\mathrm{CBD}(5,25 \% \\
\text { de CBD e } 0,2 \\
\% \text { de } \mathrm{THC})\end{array}$ & 30 DIAS & Sem efeitos & $\begin{array}{c}\text { 60.6\% dos pacientes tratado } \\
\text { s com melhoria } \\
\text { de sintomas. }\end{array}$ \\
\hline VAN, 2019 & $\begin{array}{l}\text { An experimental } \\
\text { randomized study on the } \\
\text { analgesic effects of } \\
\text { pharmaceutical-grade } \\
\text { cannabis in chronic pain } \\
\text { patients with fibromyalgia }\end{array}$ & $\begin{array}{l}22,4 \mathrm{mg} \text { de } \\
\text { THC e menos } \\
\text { de } 1 \mathrm{mg} \text { de } \\
\text { CBD. }\end{array}$ & 1 Semana & Sem efeitos & $\begin{array}{l}\text { Interações THC-CBD e o } \\
\text { papel dos sintomas } \\
\text { psicotrópicos no alívio da } \\
\text { dor. }\end{array}$ \\
\hline $\begin{array}{l}\text { MARKOVA, } \\
2019\end{array}$ & $\begin{array}{c}\text { Sativex® as add-on } \\
\text { therapy vs. further } \\
\text { optimized first-line } \\
\text { ANTispastics (SAVANT) } \\
\text { in resistant multiple } \\
\text { sclerosis spasticity: a } \\
\text { double-blind, placebo- } \\
\text { controlled randomised } \\
\text { clinical trial. }\end{array}$ & $\begin{array}{l}\text { dose oral única } \\
\text { de } 450 \mathrm{mg} \text { de } \\
\text { CBD }\end{array}$ & $\begin{array}{l}\text { DURANT } \\
\text { E } 4 \\
\text { SEMAN } \\
\text { AS }\end{array}$ & $\begin{array}{l}\text { Sem efeitos } \\
\text { relatados }\end{array}$ & $\begin{array}{l}\text { CBD proporcionou uma } \\
\text { melhoria melhor e } \\
\text { clinicamente relevante da } \\
\text { espasticidade resistente à } \\
\text { EM, em comparação com o } \\
\text { ajuste isolado de } \\
\text { medicamentos } \\
\text { antiespasticidade de } \\
\text { primeira linha. }\end{array}$ \\
\hline VAN, 2018 & $\begin{array}{l}\text { Acceptance of } \\
\text { pharmaceutical cannabis } \\
\text { substitution by cannabis } \\
\text { using patients with } \\
\text { schizophrenia. }\end{array}$ & $\begin{array}{l}\text { dose oral única } \\
\text { de } 600 \text { mg de } \\
\text { CBD (THC- } \\
\text { Pharm) }\end{array}$ & $\begin{array}{l}\text { Dose } \\
\text { única }\end{array}$ & Sem efeitos & $\begin{array}{l}\text { Sugere que uma dose única } \\
\text { de CBD em um ambiente } \\
\text { experimental pode } \\
\text { normalizar parcialmente a } \\
\text { disfunção no MTL, estriado } \\
\text { e mesencéfalo em }\end{array}$ \\
\hline
\end{tabular}




\begin{tabular}{|c|c|c|c|c|c|}
\hline & & & & & $\begin{array}{l}\text { indivíduos com CHC de } \\
\text { psicose }\end{array}$ \\
\hline $\begin{array}{l}\text { DEVINSKY, } \\
2018\end{array}$ & $\begin{array}{l}\text { Effect of Cannabidiol on } \\
\text { Drop Seizures in the } \\
\text { Lennox-Gastaut } \\
\text { Syndrome }\end{array}$ & $\begin{array}{c}20 \mathrm{mg} \text { por } \\
\text { quilograma de } \\
\text { CDB }\end{array}$ & 28 Dias & $\begin{array}{l}\text { Incluíram } \\
\text { concentraç } \\
\text { ões } \\
\text { elevadas de } \\
\text { aminotransf } \\
\text { erase no } \\
\text { fígado }\end{array}$ & $\begin{array}{l}\text { Resultou em maiores } \\
\text { reduções na frequência de } \\
\text { crises de queda do que o } \\
\text { placebo. }\end{array}$ \\
\hline $\begin{array}{l}\text { BOGGS, } \\
2018\end{array}$ & $\begin{array}{l}\text { The effects of cannabidiol } \\
\text { (CBD) on cognition and } \\
\text { symptoms in outpatients } \\
\text { with chronic } \\
\text { schizophrenia a } \\
\text { randomized placebo } \\
\text { controlled trial }\end{array}$ & $\begin{array}{l}\text { CBD oral }(600 \\
\mathrm{mg} / \mathrm{dia})\end{array}$ & 6 semanas & Sem efeitos & $\begin{array}{l}\text { Na dose estudada, o } \\
\text { aumento do CBD não foi } \\
\text { associado a uma melhora } \\
\text { nos escores em pacientes } \\
\text { ambulatoriais estáveis } \\
\text { tratados com antipsicóticos } \\
\text { com esquizofrenia. }\end{array}$ \\
\hline $\begin{array}{l}\text { CUNETTE, } \\
2018\end{array}$ & $\begin{array}{l}\text { Chronic Pain Treatment } \\
\text { With Cannabidiol in } \\
\text { Kidney Transplant } \\
\text { Patients in Uruguay }\end{array}$ & $150 \mathrm{mg} / \mathrm{dia}$ & 3 semanas & $\begin{array}{c}\text { Naúsea } \\
\text { persistente }\end{array}$ & $\begin{array}{c}\text { Durante esse } \\
\text { acompanhamento, o CBD } \\
\text { foi bem tolerado e não } \\
\text { houve efeitos adversos } \\
\text { graves. É necessário } \\
\text { estudos mais longos }\end{array}$ \\
\hline $\begin{array}{l}\text { HUNDAL } \\
2018\end{array}$ & $\begin{array}{c}\text { The effects of cannabidiol } \\
\text { on persecutory ideation } \\
\text { and anxiety in a high trait } \\
\text { paranoid group }\end{array}$ & $\begin{array}{c}\text { Canabidiol } \\
\text { oral }(600 \mathrm{mg})\end{array}$ & $\begin{array}{l}\text { Dose } \\
\text { única }\end{array}$ & Sem efeitos & $\begin{array}{l}\text { O canabidiol pode ser } \\
\text { particularmente útil para } \\
\text { ansiedade e paranóia em } \\
\text { pessoas com risco de } \\
\text { doença mental grave. }\end{array}$ \\
\hline $\begin{array}{l}\text { LICHTMAN } \\
\quad, 2018\end{array}$ & $\begin{array}{l}\text { Results of a double-blind, } \\
\text { randomized, placebo- } \\
\text { controlled study of } \\
\text { nabiximols oromucosal } \\
\text { spray as an adjunctive } \\
\text { therapy in advanced } \\
\text { cancer patients with } \\
\text { chronic uncontrolled pain. }\end{array}$ & $\begin{array}{l}\quad(\Delta 9 \text {-tetra- } \\
\text { hidrocanabinol } \\
{[27 \mathrm{mg} / \mathrm{mL}] \mathrm{e}} \\
\text { canabidiol }[25 \\
\mathrm{mg} / \mathrm{mL}])\end{array}$ & $\begin{array}{c}3 \text { dias } \\
\text { consecuti } \\
\text { vos. }\end{array}$ & Sem efeitos & $\begin{array}{l}\text { Os nabiximóis podem ter } \\
\text { utilidade em pacientes com } \\
\text { câncer avançado que } \\
\text { recebem uma dose mais } \\
\text { baixa de opióides, como } \\
\text { indivíduos com intolerância } \\
\text { precoce à terapia com } \\
\text { opióides. }\end{array}$ \\
\hline $\begin{array}{l}\text { NAFTALI, } \\
2017\end{array}$ & $\begin{array}{l}\text { Low-dose cannabidiol is } \\
\text { safe but not effective in } \\
\text { the treatment for Crohn's } \\
\text { disease, a randomized } \\
\text { controlled trial. }\end{array}$ & $\begin{array}{l}\text { CBD oral }(10 \\
\mathrm{mg})\end{array}$ & $\begin{array}{c}2 \text { vezes ao } \\
\text { dia }\end{array}$ & $\begin{array}{c}\text { Sem } \\
\text { alterações e } \\
\text { efeitos }\end{array}$ & $\begin{array}{l}\text { Falta de efeito do CBD na } \\
\text { doença de Crohn. }\end{array}$ \\
\hline $\begin{array}{l}\text { LEHMANN, } \\
2016\end{array}$ & $\begin{array}{l}\text { Experimental cannabid } \\
\text { iol treatment reduces e } \\
\text { arly pancreatic } \\
\text { inflammation in type } 1 \\
\text { diabetes. }\end{array}$ & $\begin{array}{l}5 \mathrm{mg} / \mathrm{kg} \text { de } \\
\text { CBD-dia }\end{array}$ & $\begin{array}{c}10 \\
\text { semanas }\end{array}$ & $\begin{array}{l}\text { Sem efeitos } \\
\text { colaterais }\end{array}$ & $\begin{array}{l}\text { O tratamento experimental } \\
\text { com CBD reduziu } \\
\text { marcadores de inflamação } \\
\text { na microcirculação do } \\
\text { pâncreas estudada por } \\
\text { microscopia intravital. }\end{array}$ \\
\hline $\begin{array}{l}\text { DEVINSKY, } \\
2016\end{array}$ & $\begin{array}{l}\text { Cannabidiol in patients } \\
\text { with treatment-resistant } \\
\text { epilepsy: an open-label } \\
\text { interventional trial }\end{array}$ & $\begin{array}{c}2 \text { a } 5 \mathrm{mg} / \mathrm{kg} \\
\text { por dia }\end{array}$ & $\begin{array}{c}12 \\
\text { semanas }\end{array}$ & $\begin{array}{l}\text { Diarreia, } \\
\text { náuseas e } \\
\text { convulsão }\end{array}$ & $\begin{array}{l}\text { Canabidiol pode reduzir a } \\
\text { frequência de crises e ter } \\
\text { um perfil de segurança } \\
\text { adequado em crianças e }\end{array}$ \\
\hline
\end{tabular}




\begin{tabular}{|c|c|c|c|c|c|}
\hline & & & & & $\begin{array}{l}\text { adultos jovens com } \\
\text { epilepsia altamente } \\
\text { resistente ao tratamento. }\end{array}$ \\
\hline $\begin{array}{c}\text { MANINI, } \\
2015\end{array}$ & $\begin{array}{c}\text { Safety and } \\
\text { pharmacokinetics of oral } \\
\text { cannabidiol when } \\
\text { administered } \\
\text { concomitantly with } \\
\text { intravenous fentanyl in } \\
\text { humans }\end{array}$ & $\begin{array}{l}\text { Pré-tratamento } \\
\text { de } 400 \text { ou } 800 \\
\text { mg de CBD, } \\
\text { seguido de } \\
\text { uma dose } \\
\text { única de } 0,5 \text {. }\end{array}$ & $\begin{array}{c}\text { Duas } \\
\text { sessões }\end{array}$ & $\begin{array}{c}\text { Sem efeitos } \\
\text { colaterais }\end{array}$ & $\begin{array}{l}\text { O canabidiol não exacerba } \\
\text { os efeitos adversos } \\
\text { associados à administração } \\
\text { intravenosa de fentanil. A } \\
\text { co-administração de CBD e } \\
\text { opioides foi segura e bem } \\
\text { tolerada }\end{array}$ \\
\hline $\begin{array}{c}\text { SOARES, } \\
2015\end{array}$ & $\begin{array}{c}\text { Avaliação do papel do } \\
\text { sistema canabidiol em um } \\
\text { modelo de lesão renal por } \\
\text { isquemia/reperfusão em } \\
\text { animais }\end{array}$ & $(5 \mathrm{mg} / \mathrm{kg})$ & $\begin{array}{l}\text { Dose } \\
\text { única }\end{array}$ & Sem efeitos & $\begin{array}{c}\text { O tratamento com } \\
\text { canabidiol teve um efeito } \\
\text { protetor contra a } \\
\text { inflamação e o dano } \\
\text { oxidativo em um modelo de } \\
\text { isquemia/reperfusão renal. }\end{array}$ \\
\hline
\end{tabular}

Verificou-se que os estudos enfatizam a necessidade de atualização e especialização dos profissionais que atuam no âmbito do uso terapêutico do CBD. Dessa forma, os profissionais de saúde devem estabelecer vínculos que procurem sempre aperfeiçoar seus conhecimentos, unindo a teoria e a prática e traçando um perfil que seja mais adequado na terapia medicamentosa para os pacientes que usam o CBD.

A Cannabis sativa está incluída na Lista E da ANVISA, que se refere à lista de plantas proscritas que podem originar substâncias entorpecentes e/ou psicotrópicas. O CBD, um de seus constituintes também estava listado na Lista $\mathrm{E}$, além da Lista F (BRASIL, 2015). Porém, após a utilização do CBD em tratamentos e alguns estudos em farmacologia experimental terem demonstrado que o CBD poderia ser uma importante alternativa terapêutica nas intervenções de transtornos e sintomas psiquiátricos, bem como síndromes raras que provocam convulsões nos portadores, surgiu a possibilidade de que a ANVISA pudesse considerar lícita a importação deste medicamento para o Brasil (CARDOSO, 2019).

Aguiar (2017) ressalta em sua pesquisa que há uma enorme facilidade em se fazer o tratamento com a planta fitoterápica Cannabis, já que a mesma não exige cuidados que levam a custos elevados com equipamentos sofisticados, apenas um cultivo cuidadoso. A administração em sua forma in natura em pacientes em tratamento médico e com indicação médica. Evidências preliminares sugerem que o canabidiol (CBD) pode ser eficaz no tratamento de distúrbios neurodegenerativos (BOGGS, 2018). Além do mais, através da ligação dos canabidioides com receptores existentes no Sistema Nervoso Central, estes compostos passam a agir em diversos sistemas do corpo humano (MAYER, 2015).

A análise das evidências científicas do uso do canabidiol em doenças psiquiátricas e neurológicas conclui que o CBD é um dos principais fitocanabinóides presentes na Cannabis sativa e não apresenta efeitos psicomiméticos. Além disso, o autor conclui que o "potencial terapêutico do CBD no tratamento de transtornos neurológicos e psiquiátricos é apropriado" (MATOS, 2017).

O CBD pode reduzir significativamente o uso de opióides e melhorar a dor crônica e a qualidade do sono entre os pacientes que atualmente estão usando opióides no tratamento da dor (VAN, 2019). Em 2015, a Agência Nacional de Vigilância Sanitária (ANVISA) autorizou a importação do CBD. Apesar da liberação, o preço elevado da importação torna essa substância inacessível para a maior parte da população (BENTES,2015). Guida (2019) revela que as dificuldades na obtenção de procedimentos e o alto custo do produto são os motivos determinantes para não continuar a usá-lo. Esses fatos nos questionam sobre o posicionamento do MC dentro das possibilidades terapêuticas. Seu uso não tem alcance universal, porque as atuais condições de custo permitem apenas o acesso a pessoas com um certo poder de compra dentro do espectro social. A falta de aprovação de drogas de cannabis produzidas nacionalmente pode desempenhar um papel nesse sentido. Os preconceitos e a falta de treinamento especializado sobre o assunto também contribuem para o uso informal de produtos sem o apoio profissional adequado.

Assim, a ANVISA autorizou a importação de CBD para o tratamento de doenças específicas, porém exige laudos médicos, prescrições e termos de 
responsabilidade, um processo ainda burocrático (FREITAS et al., 2019). Antes de 2015, a portaria $344 / 98$, que define os controles e as proibições de substâncias no país, regulamentou que o consumo e venda da Cannabis sativa no Brasil é ilegal (SOARES, 2016). Estudos mostram que se faz necessária a implantação de medidas regulatórias para garantir o uso adequado de medicamento à base de canabinóides, sendo assim de grande importância a presença do profissional farmacêutico para realizar o acompanhamento nesse tipo de tratamento (FREITAS et al., 2019).

Existem três principais tipos da maconha, sendo a C. sativa a mais conhecida entre a família de cannabis, sendo fisicamente a maior entre as três; seus efeitos estão pertinentes ao estímulo da mente e ao desenvolvimento de energia e criatividade. Outro tipo de maconha, experimentada como Índica, possui estrutura baixa, robusta e folhas mais largas, proporcionando baixo teor de importância psicoativa (THC); ela é famosa por seus efeitos relaxantes e calmantes, sendo ideal para combater sintomas como insônia, estresse e perda de apetite (SILVA et al., 2018).

No Brasil, a legislação regulamenta a Cannabis como uma substância ilícita, com proibição de posse, aquisição e transporte. Apenas em 2014, quando casos de crianças epilépticas tratadas com CBD começaram a ganhar visibilidade, a discussão da Cannabis medicinal no Brasil foi introduzida em pauta de autorizações da Justiça para concessão de acesso a medicamentos importados com o princípio ativo CBD. No fim do ano de 2014, o Conselho Regional de Medicina liberou o uso da Cannabis e, em 2015, a Agência Nacional de Vigilância Sanitária (ANVISA) retirou o CBD da lista de substâncias proibidas (JESUS et al., 2017).

Sabe-se que, em geral, uma dose de 200 a 300 mg por dia de CBD quando administrada em uma pessoa por curto período de tempo tem trazido resultados plausíveis, logo, a melhora do paciente, mesmo que não seja significativa, contribui para eficiência do CBD e a sua possível industrialização como medicamento (BRAGATTI, 2015).

O canabidiol tem sido usado para crises resistentes ao tratamento em pacientes com epilepsia de início precoce grave, resultando em maiores reduções na frequência de crises (DEVINSKY, 2018).

O Canabidiol apresenta uma ação inibidora contra as principais propriedades do Tetrahidrocanabinol, e vem sendo considerado importante na produção sintética do próprio THC. Ambos nunca foram isolados da Cannabis sativa em sua forma homogênea. Os dois podem ser caracterizados como antagônicos altamente competitivos, sempre buscando um superar o outro. Assim, quando o THC age proporcionando estágios de euforia, o CBD atua como bloqueador e inibidor do senso de humor. Tanto o tetrahidrocanabinol (THC) como o Canabidiol (CBD) é destacado por suas propriedades medicinais. Conforme constatado acima, os ligantes endógenos e os canabinóides, por conta de sua formulação, acabam por oferecer ação analgésica, antitumoral, aumento de apetite, redução da insônia e relaxamento muscular (MIRANDA, 2016).

De acordo com os estudos de Turner e Agrawal (2017) a intoxicação provida pela Cannabis sativa está altamente relacionada com a quantidade de dose e via de administração da mesma, estando também a quantidade causadora dos efeitos. Ademais, para os autores, entre os efeitos da intoxicação aguda, está a apresentação de alterações de percepção, euforia transitória, deficiência motora, assim como episódios de pânico, depressão e medo.

$\mathrm{Na}$ pesquisa de Oliveira (2015), alguns canabinóides possuem eficácia em torno de 200 vezes maior do que a da morfina. De acordo com ensaios clínicos, também proporcionam efeitos benéficos a pacientes impossibilitados de cura, citando-se doenças como o HIV/AIDS, câncer em fase terminal e também doenças neurológicas, como esclerose lateral amiotrófica (ELA).

Também não está claro se os efeitos do CBD persistirão após a administração a longo prazo (VAN, 2018). Na pesquisa de Souza (2017), foram encontrados artigos que trazem uma perspectiva dos novos canabinoides sintéticos. Não se sabe muito sobre eles para o uso terapêutico, no entanto, apresentam efeitos psicoativos superiores ao próprio THC e são drogas emergentes que estão sendo apreendidas por uso indevido. Atualmente, a necessidade de obter alternativas ao processo de adoecimento enfrentado pela sociedade reacendeu o ânimo por seu uso terapêutico, razão pela qual diversos estudos passaram a ser realizados nas últimas décadas sobre as propriedades farmacológicas e o potencial de uso terapêutico (LESSA, 2016).

No que se refere aos sintomas e patologias de consulta, estes apontam principalmente para o grau crônico e grave da esfera neurológica psiquiátrica, destacando a relativa insuficiência de tratamentos médicos convencionais em relação a esta (GUIDA, 2019). Considerados úteis também no tratamento da dor, náusea e epilepsia, mas podem suportar e aumentar o risco de eventos cardiovasculares (MARINELLI, 2017).

Pesquisa de Oliveira (2015) conclui que os canabinóides apresentam elevados potenciais para 
futuros regimes terapêuticos para o tratamento de diversas doenças. Os autores acrescentam que, entre os benefícios da substância para uso terapêutico, está a não ocorrência de alguns efeitos adversos como a depressão respiratória, assim como de efeitos psicoativos. Porém, maiores estudos, em especial de longo prazo, fazem-se necessários para certificação destes efeitos, assim como seu uso.

Oliveira e Lima (2016) fundamentam que o canabidiol, mais conhecido como CBD, possui habilidade antipsicótica mediante sua disponibilidade de anandamida, além de ser auxiliador na sinalização dos endocanabinóides, diante do impedimento da recaptação da anandamida. Além disso, os autores completam que o CBD pode ser utilizado no tratamento de doenças como a Epilepsia, Artrite Reumatóide, Doença de Parkinson, Doença de Alzheimer, Ansiedade e Depressão. Tais resultados corroboram a pesquisa de Oliveira (2015), que relata algumas características farmacológicas do canabidiol e ressalta que o canabidiol não possui efeitos psicoativos e uma ampla faixa de dosagem. Em contrapartida, são necessários estudos de longo prazo, pois os dados presentes na literatura não satisfazem os critérios estabelecidos para que estes compostos sejam utilizados como fármacos.

De acordo com o Protocolo Clínico e Diretrizes Terapêuticas da epilepsia, a doença está associada com a perturbação da função cerebral, possuindo como característica a recorrência de episódios de crises epiléticas, sendo estas não provocadas. A epilepsia possui fatores genéticos, metabólicos ou estruturais como causadores da doença, os quais acarretam consequências cognitivos, psicológicos, assim como também sociais, afetando âmbitos socioeconômicos dos indivíduos portadores (BRASIL, 2015). No entanto, elevadas ocorrências de convulsões intensas tornam mais grave o prognóstico do paciente (SANTOS; SCHERF; MENDES, 2019).

A pesquisa está se expandindo para o uso de canabidiol como um medicamento anticonvulsivante. $\mathrm{O}$ mecanismo do canabidiol na epilepsia pediátrica não é claro, mas acredita-se que desempenhe um papel na modulação da transmissão sináptica. As evidências de sua eficácia no tratamento da epilepsia são limitadas, mas crescentes (CHEN, 2018).

Estudos de Brucki et al. (2015) mencionam que o efeito antiepilético por uso de CBD possui comprovação, no entanto, a segurança de administração prolongada, assim como conhecimentos de propriedades farmacocinéticas, ação e interações farmacológicas com outros canabinóides, ainda se encontram confusos, necessitando de maiores esclarecimentos. Corroborando, assim, o posicionamento de Santos; Scherf e Mendes (2019), ao concluírem, em seu estudo, que se fazem necessárias maiores pesquisas com foco na resolução de tais fatores, podendo, desta forma, avançar os estudos dos usos da substância como medidas terapêuticas.

Em vista ao uso da substância para o tratamento da Esclerose Múltipla de acordo com os estudos de Giacoppo et al. (2015), onde foi utilizada uma dose de $10 \mathrm{mg} / \mathrm{kg}$ de CBD durante 14 dias, identificouse que a substância induziu efeito anti-apoptótico contra processos neurodegenerativos da esclerose múltipla. Além disso, Kozela et al. (2015), ao realizarem estudo in vitro para avaliar o mecanismo de ação dos efeitos imunorreguladores do CBD, identificaram efeito antiinflamatório, em condições autoimunes. Ademais, Costa (2017) conclui que, de acordo com os resultados de estudos já realizados, o CBD ocasiona efeito terapêutico no tratamento da esclerose múltipla. Porém, fazem-se necessários maiores estudos.

De acordo com Silva et al. (2017), ao discutir aspectos farmacológicos da substância do CDB, pesquisas identificaram que, quando administrado por via intravenosa, possui duração de 18 a 33 horas, de 2 a 5 dias quando por administração oral e de 27 a 35 horas por meio do uso do fumo. Silva et al. (2017) em seu estudo, que, a farmacocinética do CDB ocorre por meio do metabolismo de primeira passagem, sendo transformado em metabólicos para o Sistema Nervoso Central, estando entre estes o ácido 7-oic-CBD e o 7hidroxi-CBD, farmacodinâmica com afinidade pelos receptores $\mathrm{CB} 1$ e B2. A maioria dos estudos básicos mostraram um efeito positivo do CBD em comportamentos ou alterações bioquímicas (SANTOS, 2019).

\section{CONSIDERAÇÕES FINAIS}

Os estudos encontrados sobre CBD no período descrito relacionam-se a artigos de revisão. Para essa revisão os elementos levantados sugerem que o CBD pode ser um recurso terapêutico interessante no tratamento dos transtornos de ansiedade, epilepsia, depressão e esquizofrenia. Além disso, a tolerância, a toxicidade e a dependência com o uso do CBD têm sido pouco relatadas.

Com a atual revisão, percebe-se que, o foco dos estudos clínicos em humanos deve ser realizado, com período maior de observação dos efeitos em tratamentos prolongados, assim como analisado os efeitos adversos ao uso do CDB. Em estudos com animais podemos identificar que existem poucos estudos relacionando o uso do CDB em ratos doentes, grande parte está 
relacionada ao mecanismo de ação do composto.

Apesar de pequenos avanços legais terem surgidos no âmbito jurídico no Brasil, os estudos têm mostrado interesse do campus da pesquisa em ciências da saúde para a identificação de resultados significativos do uso da substância CDB encontrada na folha da planta maconha. No entanto, evidencia-se a necessidade de maiores incentivos fiscais e de pesquisas brasileiras para esta área de estudo, buscando não somente identificar seus resultados positivos, como também permitir o uso de medicamentos com a substância no Brasil.

Assim, acreditamos que as pesquisas brasileiras poderiam ser intensificadas e contribuir na comunidade científica se a legislação pertinente à importação e ao uso do CBD for amenizada. Desse modo, serão necessários amplos debates, aprofundamento das pesquisas científicas experimentais e clínicas para elucidação das propriedades terapêuticas do CBD e contribuição com a possibilidade de identificação de novas abordagens terapêuticas para o tratamento de diversas patologias. A partir do aperfeiçoamento dessa discussão, será possível a mudança de atitudes, pensamentos e leis, tudo em favor da proteção à saúde de todos os pacientes envolvidos.

\section{AGRADECIMENTOS}

A Prof ${ }^{a}$. Esp. Fabrina de Moura Alves Correia, pelas contribuições substanciais para a concepção desse artigo.

\section{REFERÊNCIAS}

AGUIAR, Fabio Alex Santana Aguiar. Uso Medicinal Para o Tratamento da Dor e Ação Neuroprotetora. 2017. 37f. Monografia (Obtenção do Título de Biomédico) Laureate International Universities, Rio de Janeiro.

ALEXANDRE, R. Canabinoides: conheça os principais compostos da maconha e suas propriedades medicinais. Disponível em https:// abraceesperanca. Com.br/2015/11/canabinoides-cobheça-os principaiscompostos-da-maconha-e- suas-propriedadesmedicinais. Acesso: 05/06/2017.

BENTES, L. Ponham as cartas na mesa e discutam essas leis: a Luta pela legislação da maconha no Brasil. Argumentum, v. 7, n. 1, p. 93- 107, 2015.

BOGGS, Douglas L. et al. The effects of cannabidiol (CBD) on cognition and symptoms in outpatients with chronic schizophrenia a randomized placebo controlled trial. Psychopharmacology, v. 235, n. 7, p. 1923-1932, 2018.

BRAGATTI, J. A. O Uso do Canabidiol em Pacientes com Epilepsia. Universidade Federal do Rio grande do Sul. Revista da AMRIGS, Porto Alegre, 59 (1): 60, jan.mar. 2015.

BRASIL. Ministério da Saúde. Protocolo Clínico e Diretrizes Terapêuticas da epilepsia. Retificada em 27 de novembro de 2015. Disponível em: http://portalsaude.saude.gov.br/images/pdf/2015/deze
mbro/01/PT-SAS-N--1319- Epilepsia-retificada.pdf. Acesso 18.02.2020.

BRUCK, Sonia M. D. Cannabinoids in neurology Brazilian Academy of Neurology. Revista Arq. NeuroPsiquiatr. vol.73 no.4, 371-374, São Paulo, 2015.

CAPANO, Alex; WEAVER, Richard; BURKMAN, Elisa. Evaluation of the effects of CBD hemp extract on opioid use and quality of life indicators in chronic pain patients: a prospective cohort study. Postgraduate Medicine, 2020, 132.1: 56-61.

CARDOSO, Simone Rodrigues. Canabidiol: estado da arte e os caminhos para a regulamentação no Brasil. 2019.

CHEN, Kerrie-Anne, et al. Cannabis for paediatric epilepsy: challenges and conundrums. Medical Journal of Australia, 2018, 208.3: 132-136.

CUÑETTI, Leticia et al. Chronic pain treatment with cannabidiol in kidney transplant patients in Uruguay. In: Transplantation proceedings. Elsevier, 2018. p. 461464.

FREITAS, A. G. P. LUCENA, Karla Camilla Lins; EDUARDO, Anna Maly de Leão e Neves; ALCÂTARA; Guizelle Aparecida de; PINHEIRO, Guilherme Junio Pinheiro. O uso do canabidiol (CBD) em doenças neurológicas: uma análise da situação no 
Brasil. Revista de Iniciação Cientifica e ExtençãoREIcEn. 2019.

GIACOPPO, Sabrina et al. A new formulation of cannabidiol in cream shows therapeutic effects in a mouse model of experimental autoimmune encephalomyelitis. Journal of Pharmaceutical Sciences. P .23-48. Oct. 2015. Disponível em: https://www.ncbi.nlm.nih.gov/pmc/articles/PMC46183 47/pdf/40199_2015_Article_131.pdf. Acesso em: 21 de Fev de 2020.

GONTIJO, Erica Cardoso; CASTRO, Geysilla Lorrany; PETITO, Anamaria Donato de Castro; PETITO, Guilherme. Canabidiol e suas complicações Terapêuticas. Revista Refacer v. 5, n. 1, 2016.

GUIDA, Julia Galzerano, et al. Cannabis medicinal como recurso terapéutico. Revista Médica del Uruguay, 2019, 35.4: 289-297.

HUNDAL, Harneet et al. The effects of cannabidiol on persecutory ideation and anxiety in a high trait paranoid group. Journal of Psychopharmacology, v. 32, n. 3, p. 276-282, 2018.

JESUS, Antônio Carlos Justo de; FERNANDES, Luis Rodrigues; ELIAS, Paloma Sampaio; SOUZA, André Ricardo Gomes de. Legalização da maconha para fins Medicinais. Revista do Curso de Direito da Universidade Braz Cubas, v. 1, n.1, maio, 2017.

KOZELA, Ewa et al. Cannabidiol, a non-psychoactive cannabinoid, leads to EGR2-dependentanergy in activated encephalitogenic T cells. p.12: 52. Mar. 2015. Disponível em: https://www.ncbi.nlm.nih.gov/pmc/articles/PMC43630 52/pdf/12974_2015_Article_273.pdf.Acesso em: 21 Fev 2020.

LEHMANN, Christian et al. Experimental cannabidiol treatment reduces early pancreatic inflammation in type 1 diabetes. Clinical hemorheology and microcirculation, v. 64, n. 4, p. 655-662, 2016.

LESSA, Marcos Adriano; CAVALCANTI, Ismar Lima; FIGUEIREDO, Nubia Verçosa. Cannabinoid derivatives and the pharmacological management of pain. Revista dor, 2016, 17.1: 47-51.

LICHTMAN, Aron H. et al. Results of a double-blind, randomized, placebo-controlled study of nabiximols oromucosal spray as an adjunctive therapy in advanced cancer patients with chronic uncontrolled pain. Journal of pain and symptom management, v. 55 , n. 2, p. 179188. e1, 2018.

Markovà J, Essner U, Akmaz B, Marinelli M, Trompke C, Lentschat A, Vila C. Sativex ${ }^{\circledR}$ como terapia complementar vs. outros ANTispásticos de primeira linha otimizados (SAVANT) na espasticidade resistente à esclerose múltipla: um ensaio clínico randomizado, duplo-cego e controlado por placebo. Int J Neurosci . 2019 Feb; 129 (2): 119-128. doi: 10.1080 / 00207454.2018.1481066. Epub 2018 Set 13.

MANINI, Alex F. et al. Safety and pharmacokinetics of oral cannabidiol when administered concomitantly with intravenous fentanyl in humans. Journal of addiction medicine, v. 9, n. 3, p. 204, 2015.

NAFTALI, Timna et al. Low-dose cannabidiol is safe but not effective in the treatment for Crohn's disease, a randomized controlled trial. Digestive diseases and sciences, v. 62, n. 6, p. 1615-1620, 2017.

MARINELLI, Lucio et al. A randomised controlled cross-over double-blind pilot study protocol on THC: CBD oromucosal spray efficacy as an add-on therapy for post-stroke spasticity. BMJ open, v. 7, n. 9, p. e016843, 2017.

MATOS, R. L. A. et al. O uso do canabidiol no tratamento da epilepsia. Revista Virtual de Química, Niterói, v. 9, n. 2, p. 786-814, 2017.

MAYER, L. S; NUNES, K. T, MARQUES. O. M, MACÊDO, C. L. Ação da Canabis e todos os custos industriais incorridos no processo produtivo terão seu reconhecimento final quando da venda dos produtos produzidossativa no combate à émese provocadas pelos anteneoplasticos. Revista: Ciênc. Saúde Nova esperança. v 13, n 1. 112. 19, de jun. 2015.

MELO, L. A.; SANTOS, A. O. O uso do Canabidiol no Brasil e o posicionamento do órgão regulador. Cadernos Ibero-Americanos de Direito Sanitário, Brasília, DF, v. 5, n. 2, p. 43-55, 2016.

MIRANDA, R. C. O Canabidiol: Seu Uso No Brasil. 2016. 25 f. Monografia (Grau de Bacharelado em Farmácia) - Faculdade de Educação e Meio Ambiente FAEMA, Ariquemes - RO.

OLIVEIRA, Kauanna Lamartine Brasil; LIMA, Thaís 
Palma Silva. Cannabis Sativa: Potencial Terapêutico. 2016. 24f. Monografia (apresentada como requisito parcial à obtenção do grau de Bacharel em Biomedicina) - Faculdade São Lucas, Porto Velho-RO.

OLIVEIRA, Maitê Thainá; PAIM, Roberta Soldatelli Pagno. O USO TERAPÊUTICO DE CANABINÓIDES EM PACIENTES PORTADORES DE DOENÇAS CRÔNICAS. 2015.

PINTO, W. S.; CIPRIANO, V. T. F. Uso terapêutico de canabinoides: perspectivas e implicações no contexto forense. Revista Acta de Ciências e Saúde, v. 1, n. 4, p. 13-30, 2015.

SANTOS, Arnóbio Barros; SCHERFB, Jackelyne Roberta; MENDES, Rafael de Carvalho. Eficácia do canabidiol no tratamento de convulsões e doenças do sistema nervoso central: revisão sistemática. Revista Acta Brasiliensis, 3(1): 30-34, 2019.

SANTOS, Rafael G.; HALLAK, Jaime EC; CRIPPA, José Alexandre S. O uso do canabidiol (CBD) no tratamento da doença de Parkinson e suas comorbidades. Revista de Medicina, v. 98, n. 1, p. 46$51,2019$.

SILVA, A. S. et al., A maconha nas perspectivas contemporâneas: benefícios e Malefícios. Revista Científica da Faculdade de Educação e Meio Ambiente. FAEMA. Ariquemes, v.9 n. 2, p. 786795 jul. dez. 2018.

SILVA, Adriana Souza da; GOMES, Jayne; PALHANO, Morgana Bonfim; ARANTES, Ana Claudia Yamashiro. A maconha nas perspectivas contemporâneas: benefícios e malefícios. Revista Cientifica da Faculdade de Educação e Meio Ambiente
- FAEMA, Ariquemes, v. 9, n. 2, p. 786-795, jul.-dez. 2018.

SILVA, Daniele Oliveira Ferreira da, et. al. O Uso do Canabidiol no Tratamento da Ansiedade. Rev Med Saude Brasilia, 6(2):255-60, 2017.

SOARES, Milena Karla. Proibicionismo e poder regulatório: uma análise do processo de classificação de substâncias. 2016. 61 f. Monografia (Requisito parcial para obtenção de grau de Bacharel em Direito) Faculdade de Direito. Universidade de Brasília, Brasília.

SOARES, Rodrigo Zon et al. Avaliação do papel do sistema canabidiol em um modelo de lesão renal por isquemia/reperfusão em animais. Revista Brasileira de Terapia Intensiva, v. 27, n. 4, p. 383-389, 2015.

SOUZA, Yago Pereira. Sínteses e Aplicações Recentes do $\Delta 9$-Tetraidrocanabinol (THC) e seus Derivados em Química Medicinal. 2017.32 f. [Monografia] Universidade Federal de São João del-Rei. São João del-Rei.

TURNER, A. R.; AGRAWAL, S. Toxicity, Marijuana. Treasure Island: StatPearls Publishing, 2017.

VAN DE DONK, Tine, et al. An experimental randomized study on the analgesic effects of pharmaceutical-grade cannabis in chronic pain patients with fibromyalgia. Pain, 2019, 160.4: 860.

VAN AMSTERDAM, Jan, et al. Acceptance of pharmaceutical cannabis substitution by cannabis using patients with schizophrenia. Harm reduction journal, 2018, 15.1: 1-4. 